\title{
Correction: A metabolomic and pharmacokinetic study on the mechanism underlying the lipid- lowering effect of orally administered berberine
}

Cite this: Mol. BioSyst., 2015, 11,664

DOI: $10.1039 / \mathrm{c} 4 \mathrm{mb} 90042 \mathrm{a}$

www.rsc.org/molecularbiosystems

\author{
Shenghua Gu, ${ }^{\text {abc }}$ Bei Cao, ${ }^{\text {ad }}$ Runbin Sun, ${ }^{a}$ Yueqing Tang, ${ }^{\mathrm{b}}$ Janice L. Paletta, ${ }^{\mathrm{e}}$ \\ Xiaolei Wu, ${ }^{\text {b }}$ Linsheng Liu, ${ }^{a}$ Weibin Zha, ${ }^{a}$ Chunyan Zhao, ${ }^{a}$ Yan Li, ${ }^{\text {b }}$ \\ Jason M. Ridlon, ${ }^{e}$ Phillip B. Hylemon, ${ }^{e}$ Huiping Zhou, ${ }^{e}$ Jiye Aa*a and Guangji Wang ${ }^{a}$ \\ Correction for 'A metabolomic and pharmacokinetic study on the mechanism underlying the lipid-lowering \\ effect of orally administered berberine' by Shenghua Gu et al., Mol. BioSyst., 2015, DOI: 10.1039/c4mb00500g.
}

The spellings of two of the authors' names were incorrect. Jason M. Radlon should read Jason M. Ridlon and Xiao-Lei Wu should read Xiaolei Wu. In addition, the address for Janice L. Paletta was incorrectly given as 'Center for Drug Safety Evaluation and Research, Shanghai University of Traditional Chinese Medicine, China'. The correct address for this author is 'Department of Microbiology \& Immunology, Virginia Commonwealth University, Richmond, USA'. The full author list should be Shenghua Gu ${ }^{\text {abc }}$, Bei Cao ${ }^{\text {ad }}$, Runbin Sun ${ }^{\mathrm{a}}$, Yueqing Tang ${ }^{\mathrm{b}}$, Janice L. Paletta ${ }^{\mathrm{e}}$, Xiaolei $\mathrm{Wu}^{*^{\mathrm{b}}}$, Linsheng Liu ${ }^{\mathrm{a}}$, Weibin $\mathrm{Zha}^{\mathrm{a}}$, Chunyan $\mathrm{ZhaO}^{\mathrm{a}}$, Yan $\mathrm{Li}^{\mathrm{b}}$, Jason M. Ridlon ${ }^{\mathrm{e}}$, Phillip B. Hylemon ${ }^{\mathrm{e}}$, Huiping Zhou ${ }^{\mathrm{e}}$, Jiye Aa ${ }^{*^{\mathrm{a}}}$ and Guangji Wang ${ }^{\mathrm{a}}$

The Royal Society of Chemistry apologises for these errors and any consequent inconvenience to authors and readers.

\footnotetext{
${ }^{a}$ State Key Laboratory of Natural Medicines, Key Laboratory of Drug Metabolism and Pharmacokinetics, Jiangsu Key laboratory of drug design and optimization, China Pharmaceutical University, Nanjing 210009, China. E-mail: jiyea@cpu.edu.cn

${ }^{b}$ College of Engineering, Peking University, Beijing 100871, China.E-mail:xiaolei_wu@pku.edu.cn

${ }^{c}$ Center for Drug Safety Evaluation and Research, Shanghai University of Traditional Chinese Medicine, China

${ }^{d}$ Nanjing Drum Tower Hospital, the Affiliated Hospital of Nanjing University Medical School, Nanjing, China

${ }^{e}$ Department of Microbiology \& Immunology, Virginia Commonwealth University, Richmond, Virginia, 23298, USA
} 